\title{
COMPREHENSIVE APPROACH TO EVALUATION OF DEGRADATION IN CHOSEN PARTS OF ENERGY EQUIPMENT
}

\author{
Sylvia Kusmierczak, Tomas Majzner \\ Jan Evangelista Purkyně University in Ústí nad Labem, Czech Republic \\ e-mail: kusmierczak@fvtm.ujep.cz
}

\begin{abstract}
The individual construction elements are exposed to varying forms of loading, which correspond to the symptoms of degradation. In the analysis of degradation it is necessary to choose an approach that will lead to determine the actual causes of its origin. For determining the causes of degradation of objects currently wide variety of methods and equipment are available. It is necessary often to have in mind the economic viewpoint of the used method of analysis. It follows effort to create an approach for the educational process that passes information to students about choosing the methodologies and equipment. The present paper theoretically and practically introduces the possibility of a comprehensive approach that gives students the skills to detect the causes of degradation in selected parts of the energy equipment. This is an approach and examples for students studying technical fields. Students can apply this information during their studies in solving practical problems in the subjects such as Materials Science, Wear, Degradation of Metal Materials.
\end{abstract}

Keywords: degradation, energy equipment, approach.

\section{Introduction}

The design elements and technical objects are loaded with a number of factors during their lifetime, which leads to decrease of the functional properties and to degradation, resulting in failure of the material $[1 ; 2]$. For users, this represents in the best case economic losses, in the worst case there is danger to life. These, as well as less catastrophic impacts, lead to failure on components and units, leading to the need to predict the material behaviour by operational conditions [3; 4].

The technical object can be characterized as any technical portion structural member, which may be isolated or forms a part of the assembly. Technical object (TO) has a definite geometric shape, morphology, size, chemical, physical and mechanical properties [5; 6].

The state quantities of TO are related to the action on the object, object properties, bond structures and manifestations of the object. They depend on the nature of state variable change over time, may extend these changes continuously or in steps. Some of these changes may cause such a condition, which is inadmissible for another function. Unacceptable conditions may occur during manufacture, handling, operation or repair. These states are in technique known as limit states [5-8].

According to [8] the limit state is a state of the material or component, in which the result of small changes in external or internal factors, about a certain (critical) size and time of their action material, component by jumping lose, functional and utility properties, or gradual change of function and properties of materials, reaches a critical value. Limit states that may be in operation occur, and if not removed, will cause degradation or even crash of the object. Degradation technical object can be defined as a permanent, functionally undesirable change of the surface dimension of the technical object resulting from a wear mechanism [9]. According to [10], the term degradation (damage) means the changes in the properties of the material during its use making this material gradually to become unsuitable for a given application. The degradation process can be triggered by factors, which under the operational load can lead to combination. Some are caused by mechanical loading of the material (plastic deformation, fracture, fatigue, wear), others by mechanical forces applied, also corrosive action, etc. But always undesirable change appears in the original characteristics that can be deformation, structural or include surface geometry, which leads to an overall reduction in the quality of the TO. TO degradation can occur by changing the material characteristics, the occurrence of cracks or stress changes at or below the surface of the material of the TO, etc.

Consequences of degradation processes in technical objects (TO) not only lead to undesirable changes in the properties and the consequent shortening life cycle of TO, but also to economic losses and threats to user safety subassembly. Therefore, it is necessary to apply a systemic approach for evaluating the degradation processes.

Proposal of the methodology for the comprehensive approach to degradation processes is focused in this work on selected technical objects made from metal materials. This belongs to the group of 
materials having common characteristics and response during external action on TO. The proposed methodology for comprehensive approach to degradation processes $[10 ; 11]$ has the following main steps:

- location and description of the manifestations of degradation TO,

- theoretical analysis of internal and external factors of TO,

- description of the actual state of TO and the actual conditions in which it was issued,

- comparison of theoretical factors with real factors - to determine the factors that may cause the limit states and degradation processes TO,

- evaluation of degradation TO - TO analysis using microscopy techniques, destructive and non-destructive testing [12-15],

- processing of results, determination of the causes of degradation TO,

- conclusions for preventing degradation processes TO, respectively reducing of TO degradation $[3 ; 4 ; 7 ; 10]$.

This paper aims to show a comprehensive approach in the educational process, which conveys information to students for selecting the methodologies and equipment. The present article, therefore, theoretically and practically introduces the possibility of the comprehensive approach, so that students acquire the skills to uncover the causes of degradation in selected parts of the energy equipment.

\section{Materials and methods}

An example will follow for students of the process and the use of adequate methodologies for assessing the causes of degradation. Metallographic procedures using light and electron microscopy are often used in the practical solution of degraded objects. These adequately reveal the causes of degradation manifestations. A comprehensive approach to evaluating degradation of parts of the power equipment is presented by two technical objects. In the paper they are identified as a technical object number 1 and 2 .

\section{Technical object 1 - TO 1}

Location and description of the manifestations of degradation TO: occurrence of diagonal cracks, cohesive failure of the outer surface of the pipe.

Description of the actual state of TO: for analysis of thesample a specimen was taken from tubes $\varnothing 32 \times 6 \mathrm{~mm}$, material of the pipe CSN 415020.1 , i.e. steel shall be normalizing. The maximum permissible temperature of the tube wall load is $420^{\circ} \mathrm{C}$, cca 20 years of operation.

Theoretical analysis of internal and external factors: Internal factors: material - microstructure heat treatment, inclusions, surface - roughness geometry technology - casting method and forming of tubes. External factors: pressure, temperature, corrosion loading, wear and tear.

\section{Evaluation of degradation TO}

Visual evaluation: on the surface: there was a cohesive failure with a large number of diagonal oriented cracks. The increased roughness, surface geometry and distinct uneven distribution of corrosion products are shown on the tubes, Fig. 1. The damaged inner tube surface is shown in Fig. 2. The crack was initiated on the outside surface, spreading the material towards the inner diameter of the pipe. Even this surface was attacked unevenlyby distributed corrosion products.

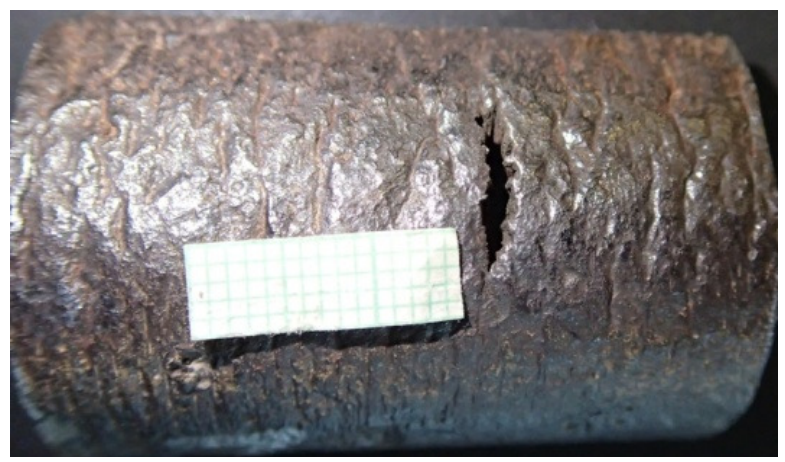

Fig. 1. TO 1, outer surface of the tube 
a)

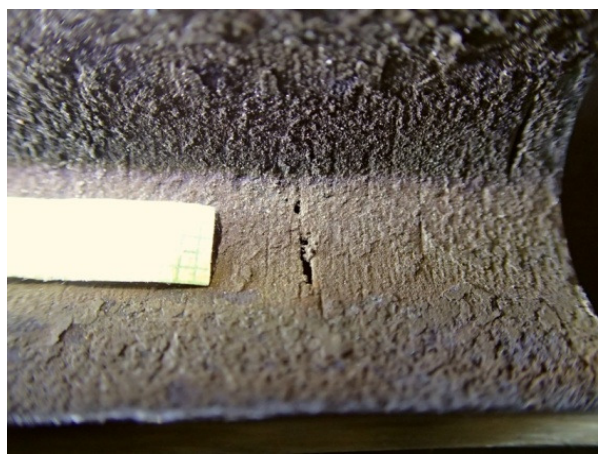

b)

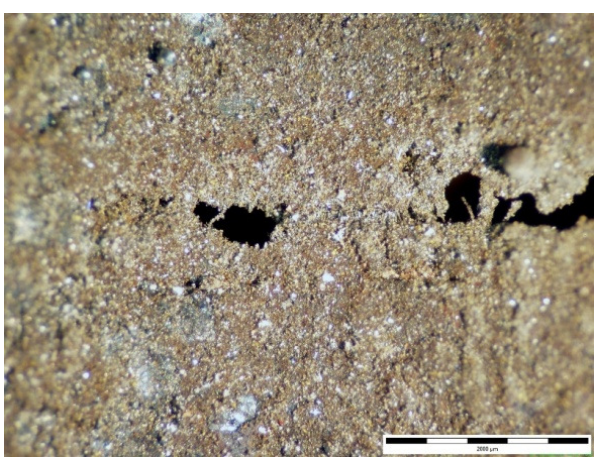

Fig. 2. TO 1, inner surface of the tube: $a$ - crack; $b$ - detail

Chemical analysis: chemical analysis was performed by spectrometry. The declared values observed were compared with the standard material to verify the quality of the tube material. The results of the analysis are show in Table 1, including the specified values, which correspond to the declared material according to the ČSN 41 5020.1.

Table 1

TO 1, element content in steel ČSN 415020.1

\begin{tabular}{|c|c|c|c|c|c|c|c|c|c|}
\hline Elements & $\mathbf{C}$ & $\mathbf{M n}$ & $\mathbf{S i}$ & $\mathbf{P}$ & $\mathbf{S}$ & $\mathbf{C r}$ & $\mathbf{N i}$ & $\mathbf{M o}$ & $\mathbf{V}$ \\
\hline TO 1 & 0.20 & 0.59 & 0.26 & 0.02 & 0.02 & 0.21 & 0.07 & 0.32 & 0.03 \\
\hline \multirow{2}{*}{ ČSN 41 5020 } & 0.12 & 0.50 & 0.15 & $\max$. & $\max$. & - & - & 0.25 & - \\
& 0.20 & 0.80 & 0.45 & 0.04 & 0.04 & - & & 0.35 & - \\
\hline
\end{tabular}

Microscopic analysis: before the microscopic analysis the pipe samples were metallographically prepared and subsequently etched.

The microstructure of the tube material is formed of ferritic and pearlitic grains, which have a non-uniform size, Fig. 3. It does not match the required heat treatment, namely the state after normalization annealing.

a)

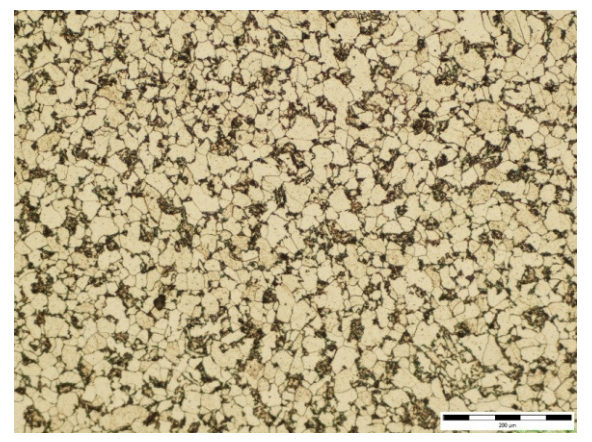

b)

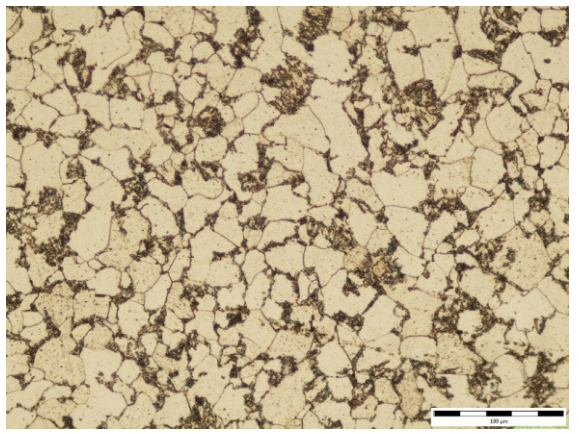

Fig. 3. TO 1, microstructure, cross sectional view of, etching: a - ferritic-pearlitic grains; $b$ - detail

Type breach of the outer and inner surface of the tubes is shown in Fig. 4. The depth of corrosive attack on the outer surface of the material achieves to half the wall thickness. The outer surface of the pipe thus exhibits corrosion attack and exhibits cracks, which are oriented perpendicularly to the propagation direction of the medium in the pipe. The transversely extending cracks are likely to have originated in the corrosive stress conditions in the pipe wall caused by temperature changes significantly when spraying the walls with boiler water. Cracks are tipped with sharp notch, which is covered with corrosion products. In locations cracks occur during pipe expansion and stress concentrations can be expected that the areas thus damaged will cause leakage.

On the inner surface corrosion attack is very small and the maximum depth is max. $0.2 \mathrm{~mm}$. Degradation of the inner surface of the tube was induced by corrosive attack in the form of pitting, Fig. 4 b. 
a)

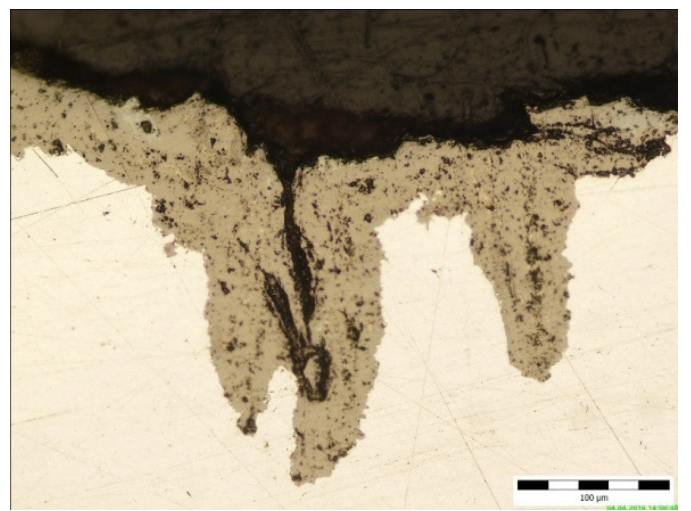

b)

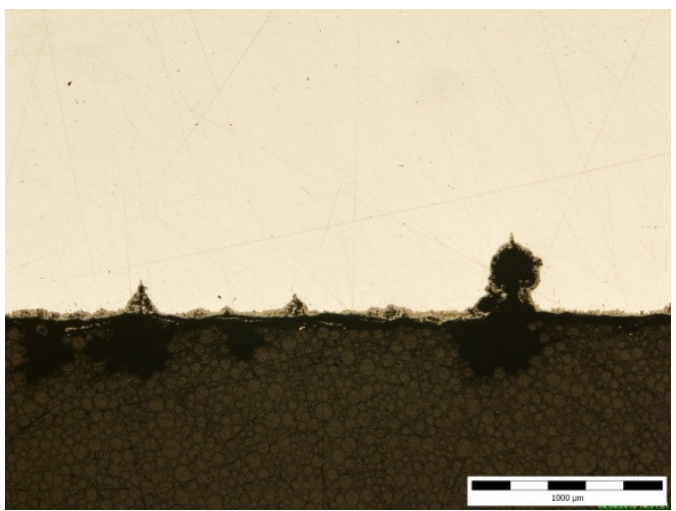

Fig. 4. TO 1, details of corrosive attack: $a$ - the outer surface; $b$ - the inner surface

\section{Technical object 2 - TO 2}

Location and description of the manifestations of degradation TO: gaping longitudinal crack in the medium flow direction. The sample was taken from the evaporator in the pipe bend, Fig. 5.

Description of the actual state of TO: the tube evaporator dimensions $\emptyset 32 \times 6 \mathrm{~mm}$, the material of CSN 415020.1 . Operating conditions: pressure $20 \mathrm{MPa}$, design temperature of the tube wall $420^{\circ} \mathrm{C}$, medium temperature $315-370{ }^{\circ} \mathrm{C}$ and around 20 years of operation.

Theoretical analysis of internal and external factors: Internal factors: material microstructure, heat treatment, inclusions, surface - roughness geometry technology - casting method and forming pipe. External factors: pressure, temperature, loading corrosion, wears.

Visual evaluation: The pipe surface was covered with thick layers of slag and all baked. Cleaning surface was seen wrinkling in the longitudinal bending, the crack openings have length $50 \mathrm{~mm}$, width $20 \mathrm{~mm}$, Fig. 6 . The wall thickness of the original size of $6 \mathrm{~mm}$ changed in the location of the interruption on the surface cohesion on $1.5 \mathrm{~mm}$, Fig. 7.

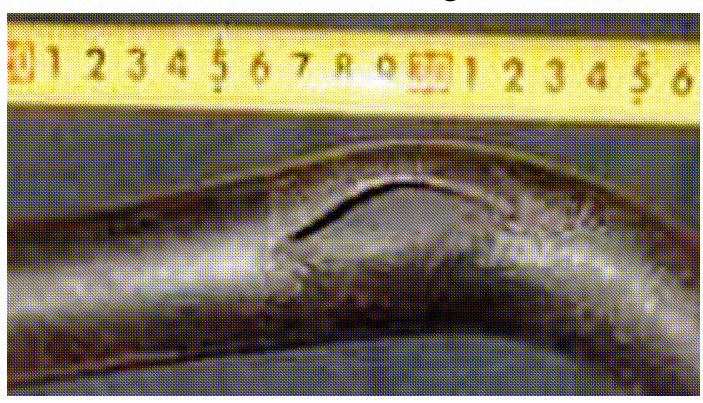

Fig. 5. TO 2

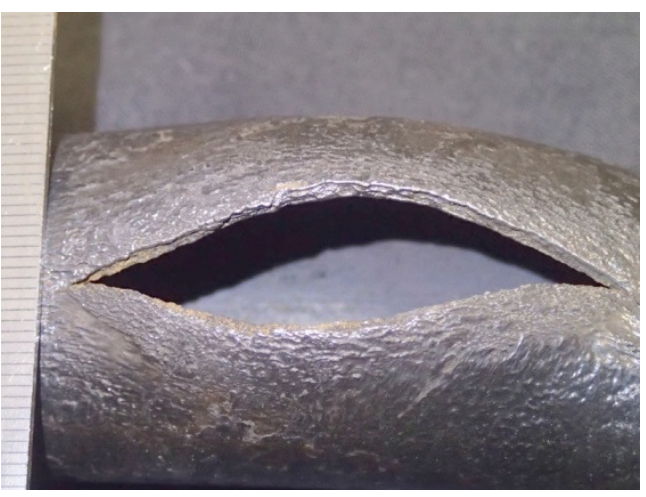

Fig. 6. TO 2, damaged pipe

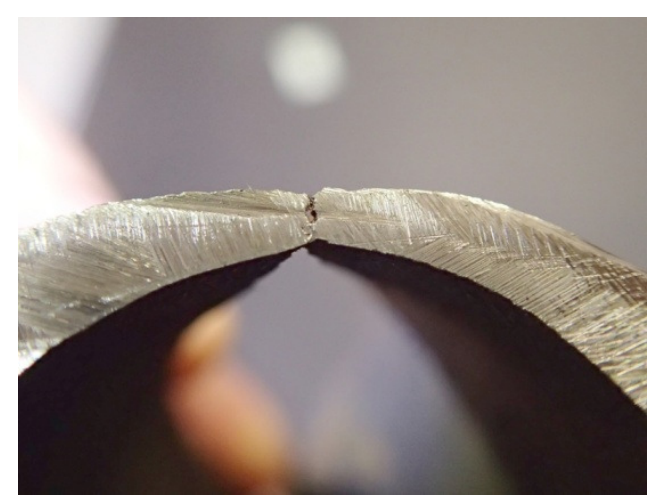

Fig. 7. TO2, changing thickness of pipe

Chemical analysis: Table 2 shows the results of analyzes including the specified values, which correspond to the declared material according to the ČSN 415020.1. 
TO2, element content in steel ČSN 415020.1

Table 2

\begin{tabular}{|c|c|c|c|c|c|c|c|c|c|}
\hline Elements (hm. \%) & C & Mn & Si & P & S & Cr & Ni & Mo & V \\
\hline TO2 & 0.13 & 0.72 & 0.30 & 0.02 & 0.01 & 0.20 & 0.06 & 0.31 & 0.02 \\
\hline ČSN 41 5020 & 0.12 & 0.50 & 0.15 & $\max$. & $\max$. & - & - & 0.25 & - \\
& 0.20 & 0.80 & 0.45 & 0.04 & 0.04 & & & 0.35 & \\
\hline
\end{tabular}

a)

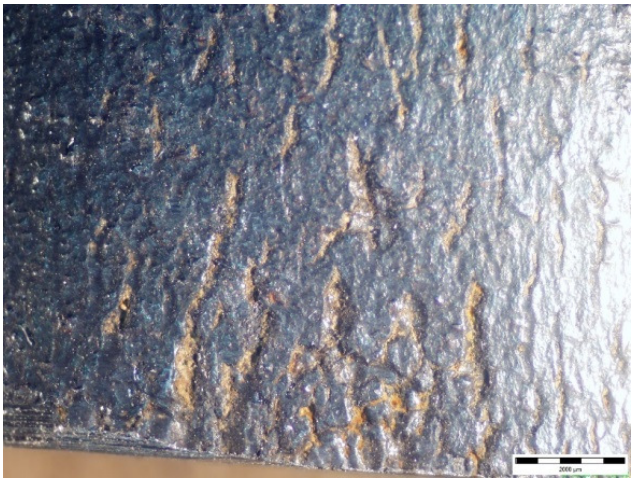

b)

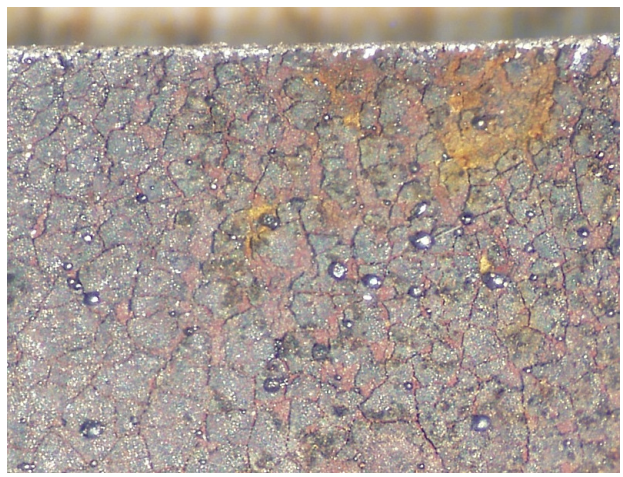

Fig. 8. TO2, tube surface: $a-$ the outer surface; $b$ - the inner surface

Microscopic analysis: Fig. 8 shows the details of the outer and inner wall surfaces of the pipe cracked protective oxide layer. The pipe cross-sectional detail of the damaged oxide layer outside and inside surface is shown in Fig. 9. The thickness of the oxide layers is $0.25 \mathrm{~mm}$ on the outer surface, $0.1 \mathrm{~mm}$ on the inner surface.

a)
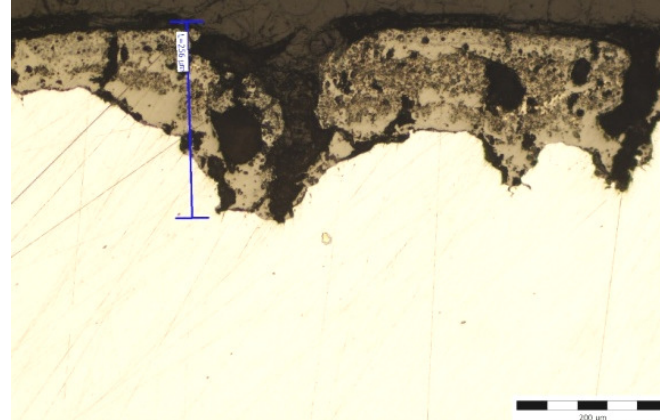

b)

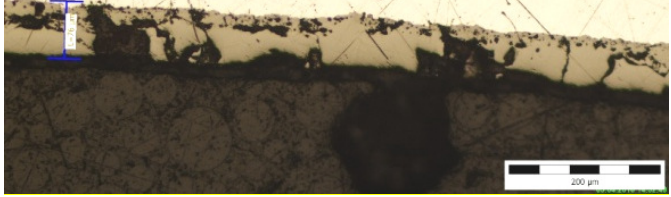

Fig. 9. TO2, cross sectional view of, the oxide layer: $a$ - the outer surface; $b$ - the inner surface

The pipe material has a ferrite-pearlite structure (Fig. 10 a) with a heterogeneous grain sizes. The area of destruction (Fig. $10 \mathrm{~b}$ ) is characterized by significant structural degradation consisting in breaking the original composite structure to ferrite unevenly distributed carbides. There are cracks visible in cavities at grain boundaries, which are the side effect of creep.

a)

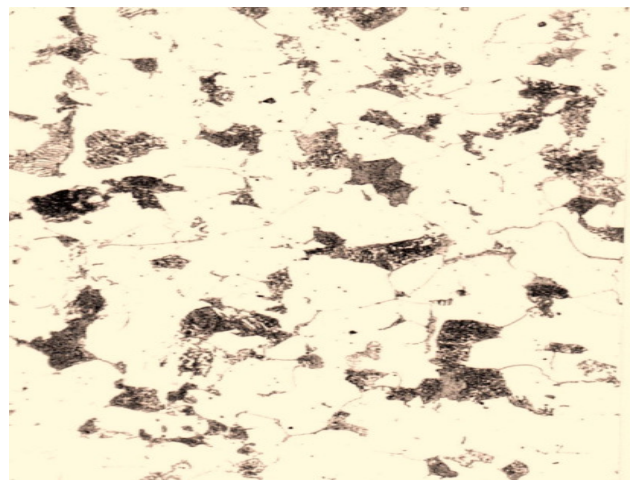

b)

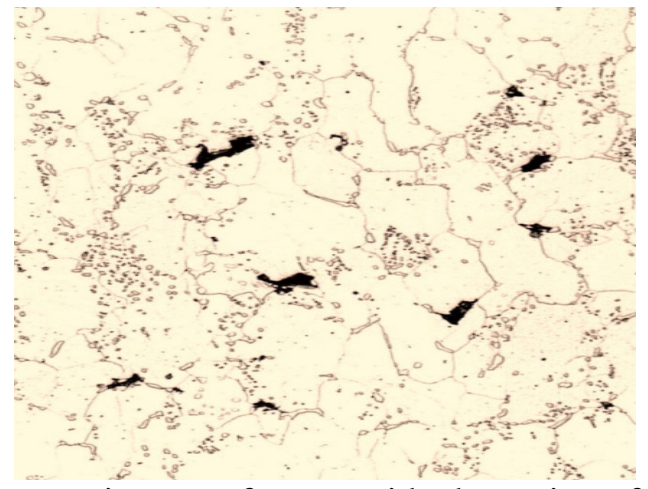

Fig. 10. TO2, microstructure, cross sectional view: a - inner surface, outside the region of degradation, magn. 500x; $\mathrm{b}$ - inner surface, in the region of degradation, magn. 500x 


\section{Discussion}

The internal and external factors were determined for TO 1 that may cause degradation of the object. Based on the analysis it can be concluded that the object damage occurs due to the action of stress and corrosion. As a probable cause of this type of damage appears insufficient dilation and cyclic loading, caused by bad functioning of the cleaning device. In this it caused signs of degradation mainly by external factors - stress and corrosion loading. Recommendation: to remove the cause of degradation of the object, i.e. carry out inspection of adjustment of the water sprayer walls of the combustion chamber.

TO 2: based on analyzes it can be concluded that the object was damaged due to excessive exposure to stress in the increased thermal stress with a typical course of the crack. Increased thermal stress can be caused by insufficient flow of the cooling medium. In this case it caused degradation signs of external factors - the tension and exceeding the allowed limit for heat stress. Recommendation: places where the pipeline at present has degradation are not possible to locate and even predict the formation of the actual degradation. This is often associated with reductions in the flow of the cooling medium, a result of which there is a significant temperature increase above the acceptable values resulting in the creep of the material and, in the worst case, appearance of cracks and the formation of fracture surfaces. Generally, it is necessary to inspect the flow of the medium in the chamber, from which the water-steam mixture is supplied to the exchanger tubes.

\section{Results and conclusions}

The paper presented a comprehensive approach by the search of the causes of degradation of selected technical objects from the field of energy equipment. This includes a set of processes that lead to determine the actual causes of degradation processes. The first step is a description of the problem situation, which contains a set of information about what is really a technical object exposed. Before analysis is it necessary to analyze the internal and external factors, which could theoretically give rise to degradation processes. The results of this analysis narrow the selection tests, which will be needed to ensure a relevant result to determine the actual causes of degradation of the technical object. The next step is to perform selected analyzes, processing of results, their discussion, the determination of the causes of degradation and especially the recommendations on what to do in order to avoid degradation of, or slowed down the degradation process to an economically acceptable level, but always with regard to the safety of the technical object.

This process teaches students to look at the analyzed object comprehensively, that is, track it since the creation of the work piece of the object to the state where degradation processes started. And also to use methods of research materials that will lead to definite results. The outputs of the analyzes of the objects that have the same start-inducing degradation processes, can serve as a portfolio of boundary conditions for simulation creation of degradation processes in the load environment.

\section{Acknowledgement}

The authors are grateful for the support of grant EDIMARE CZ.1.05/4.1.00/11.0260.

\section{References}

1. Gasparjans A., Terebkovs A., Ziravecka A. Early diagnostics of real time technical condition of steel shafts. Engineering for Rural Development, Vol. 2016-January, 2016, pp. 1316-1323.

2. Kusmierczak S., Briza T. Degradation of Coatings in Car Repair. Engineering for Rural Development. Vol 12, 2012, pp. 262-266.

3. Savinyh P., Nechaev V., Nechaeva M., Ivanovs S. Motion of grain particle along blade of rotor fan of hammer crusher. Engineering for Rural Development, 2016, pp. 1072-1076.

4. Kuśmierczak S., Kraus P. Analysis of the causes of degradation of surface-treated sheet metal of part the lift. Internation Multidisciplinary Conference, IMD 2013. The 10th edition, Proceedings, May 22nd-24th, 2013, Baia Mara, Romania, Nyíregyháza, Hungary, 2013, pp. 83-86.

5. Janicek P. System approach for selected fields of technology. Search contexts. CERM, 2007, s. 1234, ISBN 978-80-7204-556-3, ISBN 978- 80-214-3546-9. Original in Czech.

6. Stodola J., Peslova F., Krmela J. Wear of machine parts. 2008, 197 s., ISBN 978-80-7231-552-9. (In Czech) 
7. Collins J. A. Failure of Materials in mechanical Design, Analysis, Prediction, Prevention. New York, J. Wiley and Sons, 1981.

8. Puskar A. Limited states of materials and components. Veda, 1989, s. 304, ISBN 80-224-0020-3. (In Czech).

9. Vojtech D. Materials and their limit states. Publishing VSCHT, 1. vyd., 212 s., ISBN 978-807080-741-5. (In Czech).

10. Kusmierczak S. Methods of evaluation degraded parts. Engineering for Rural Development, Vol 14(January), 201, pp. 790-794.

11. Naprstkova N., Michna S. Identification and evaluation of machining product defects in teaching of students at college. Engineering for Rural Development, 2013, pp. 569-572.

12. Kusmierczak S. Usage of technical equipment in teaching technical subjects. Engineering for Rural Development, Vol 14 (January), 2015, pp. 748-752.

13. Novotny J., Jaskevic M. Morphology and producing of TiO2 particles. Engineering for Rural Development, Volume 2016-January, 2016, pp. 1218-1223.

14. Valasek P., Chocholous P., Muller M. Mechanical properties of thermal insulating sandwich materials. Engineering for Rural Development, Volume 2016-January, 2016, pp. 324-328.

15. Kusmierczak S. The usage of confocal laser microscope by solving student projects. International Multidisciplinary Conference. 9th edition, 19-21 May, 2011, Baia Mare - Nyiregyhaza, Romania - Hungary, pp. 149-152. 